\title{
DEMOCRACIA Y REFORMA ECONOMICA EN ARGENTINA (1983-1995): ¿DOCE AÑOS NO ES NADA?
}

\author{
Antonio Camou*
}

"Sentir que es un soplo la vida, que veinte años no es nada..." Volver, tango de Carlos Gardel y Alfredo Lepera.

\section{PRESENTACION}

A muchas personas, dentro y fuera de la Argentina, les ha llevado una vida comprender al peronismo; ahora, para complicar las cosas, les ha tocado en suerte entender al menemismo. La comprensión del fenómeno se hace aún más difícil si nos demoramos en las declaraciones destempladas o en los gestos estrafalarios a que nos tiene acostumbrados el presidente Menem, si nos limitáramos al comentario frívolo o al detalle farandulesco. Ciertamente, no es fácil separar la controvertida personalidad del mandatario argentino de las profundas transformaciones sociales, económicas y políticas que ha liderado en el país en los últimos años. Pero sin ese necesario esfuerzo de distanciamiento corremos el riesgo de confundir lo accidental con lo necesario, lo esencial con lo anecdótico.

Después de cinco elecciones ganadas con claridad (1989, 1991, 1993, 1994 y las más recientes, efectuadas el 14 de Mayo de 1995) (1), el menemismo está marcando a fuego la convulsiva Argentina de fin de siglo. Desde las primeras presidencias de Perón (1946-1955), nadie había cambiado tanto la Argentina en tan poco tiempo; después de Menem, y por muchos años, el país seguirá transitando por las huellas trazadas por el pintoresco caudillo riojano.

Pero las últimas elecciones, más allá de su resultado circunstancial (2), poseen además una importancia histórica

* Sociólogo argentino (1961), Master en Ciencias Sociales de la FACULTAD LATINOAMERICANA DE CIENCIAS SOCIALES (FLACSO), Sede Académica de México. Actualmente integra el Programa de Doctorado en Ciencias Sociales de dicha institución.

1. Desde Octubre de 1983 se realizaron en la Argentina ocho elecciones generales: tres elecciones presidenciales (1983, 1989 y 1995), cuatro elecciones intermedias para la elección de legisladores nacionales y en algunos casos gobernadores de provincia $(1985,1987,1991,1993)$ y una elección para la reforma constitucional (Abril de 1994). Salvo la elección presidencial de 1983, que llevó al poder a Raúl Alfonsín, y la elección intermedia de 1985, ambas ganadas por el Partido Radical a nivel nacional, el resto de las contiendas electorales las ganó el peronismo.

2. Los datos finales le otorgaron la victoria a Menem por el $49.8 \%$ contra e $29.2 \%$ de José Octavio Bordón (lider del Frente para un Pais Solidario, FREPASO) y el $17 \%$ del candidato radical Horacio Masaccessi. digna de atención. Desde 1928, cuando el candidato radical Hipólito Yrigoyen sucedió en el cargo a su correligionario Marcelo T. de Alvear, no se producía en la Argentina una tercera elección democrática consecutiva para la renovación de un presidente constitucional. En las casi siete décadas transcurridas desde entonces el país se convirtió en el laboratorio perfecto para estudiar la inestabilidad política, la creciente conflitividad social y el comportamiento irregular de una economía que en los últimos veinte años se volvió francamente errática. En esta furiosa historia contada por un loco, en la que debemos incluir una guerra perdida contra una potencia de la OTAN en 1982, miles de muertos y desaparecidos durante los años setenta, y un episodio hiperinflacionario que llevó el índice de precios -en el último año del gobierno alfonsinista- a una cifra de cuatro dígitos, hay que buscar algunas de las raíces más profundas de este nuevo triunfo de Menem. Un triunfo que completa doce años, y no es poco, de recuperada vida democrática.

En este trabajo me propongo dos objetivos. En primer lugar, después de presentar un apretado resumen que va de Alfonsín a Menem, sintetizo lo que creo fueron los móviles fundamentales del apoyo electoral recibido por el peronismo en las últimas elecciones, cuyo eje básico hay que buscarlo en el éxito alcanzado por el plan económico de estabilización. En segundo término, examino los cambios políticos y económicos experimentados por la Argentina en la última década en una perspectiva comparativa con el resto de América Latina. En particular, analizo la vinculación entre variables político-institucionales y reforma económica, tratando de ubicar los cambios político-económicos acontecidos en la Argentina en el marco de la literatura académica sobre el ajuste. El núcleo de mi argumento apunta a señalar que las condiciones políticas e institucionales que le permitieron a Menem llevar adelante con dramática rapidez la fase "negativa" del ajuste, y que se tradujeron en un apoyo político manifiesto por parte de vastos sectores de la población, quizá se han vuelto hoy un escollo para el desarrollo de la última etapa de la reforma económica, la que se refiere a la reestructuración del perfil productivo del país. 


\section{DE ALFONSIN A MENEM}

Cuando Raúl Alfonsín asumió la presidencia en diciembre de 1983 el país enfrentaba dos gravísimos problemas a resolver: el de una larga crisis económica y el de la consolidación democrática frente al ingobernable actor militar. Casi dos años después de asumir el poder, el alfonsinismo descubrió que el primer asunto escondía un dilema fatal: la sana gestión de la economía requería medidas drásticas que terminarían por minar las bases electorales del partido en el poder; y ésta fue, precisamente, una de las razones principales que llevaron a la administración alfonsinista a optar, a mediados de 1985, por un enfoque "heterodoxo" del ajuste económico, el cual terminó naufragando a finales de 1988. En el segundo punto, a su vez, se abría un entuerto crucial para la transición: cómo subordinar efectivamente los militares al poder constitucional mientras al mismo tiempo se respondia a las demandas de justicia por las flagrantes violaciones a los derechos humanos acontecidas durante la última dictadura (1976-1983).

En términos generales, y después de muchas y desconcertantes vueltas, Alfonsín dejó esos dos problemas igual, o incluso peor, de lo que estaban cuando asumió. Menem enfrentó ambas cuestiones con extrema decisión y escaso margen. En el primer caso, y sobre esto ha corrido ya mucha tinta, optó por el rumbo que nos hemos acostumbrado a calificar como "neoliberal". En el marco de las tendencias dominantes de la economía mundial, pero con el fanatismo propio de los conversos recientes, aplicó a rajatabla las recetas de un remedio conocido. Sobre la base de la crisis hiperinflacionaria de mediados de 1989, Menem logró galvanizar una mayoría social consistente que le permitió instrumentar -con apoyo popular- las medidas que ningún elenco liberal había podido imponer en la historia argentina reciente: apertura comercial, orientación exportadora, austeridad fiscal, disciplina monetaria, menor regulación pública de los mercados y un programa de privatizaciones rápido, total, políticamente eficaz, desordenado y en muchos casos escandaloso.

En el segundo tema pactó con los militares un intercambio oscuro: les concedió el indulto por las violaciones a los derechos humanos a cambio de su subordinación al poder civil. La ecuación de este último pacto ha sido juzgada como éticamente indigna y reprobable; desde el punto de vista político, sin embargo, los resultados están a la vista. En las tres sublevaciones militares que debió atravesar el gobierno radical (una en 1987 y dos en 1988), Alfonsín nunca pudo contar con una fuerza leal dispuesta a reprimir a los sediciosos. En el único levantamiento militar que tuvo que enfrentar el gobierno peronista (diciembre del '90), Menem contó con las fuerzas suficientes como para descabezar, sangrientamente incluso, a los desubicados levantis$\cos (3)$.

La manera de enfrentar esos núcleos duros de la política argentina de los ochenta define claramente el "estilo de go-

3. Un tratamiento detallado y actualizado del periodo que va de Alfonsin a Menem se encontrará en el excelente libro de Carlos H. Acuña (comp.), La Nueva Matriz Politica Argentina, Buenos A.ires, Nueva Visión, 1995, especialmente los capitulos 4, 6 y 8 . bierno" de Menem: improvisado, pragmático, inescrupuloso, directo, efectivo. El politólogo norteamericano Samuel P. Huntington recordaba hace muchos años un lugar común de la política democrática: las elecciones se ganan con votos, pero las sociedades se gobiernan mediante la articulación de factores de poder. El asunto moverá a muchos a la indignación o a la resignada queja, pero no puede ser motivo de ignorancia. Desde que ganó las elecciones presidenciales en 1989, y en el clima incendiario de la hiperinflación, Menem se abocó a cimentar una alianza que hiciera gobernable el país. Después de seis años de haber asumido el poder puede decirse, para bien y para mal, que lo ha logrado. En las últimas elecciones generales una mayoría consistente del país entendió que por ahora $-\mathrm{y}$ mientras tanto- Menem es la única opción creíble de gobierno, el modelo factible, el rumbo consistente.

El gobierno justicialista (peronista) llegó a las elecciones del 14 de mayo de 1995 con las palmarias cifras de sus éxitos, sustentadas en una serie de meteóricas transformaciones estructurales, pero también con el acrecentado rosario de sus adeudos. Después de que la economía argentina sufriera un retroceso del PIB per cápita de $-21.2 \%$ entre 1981 y 1990, la dupla Menem-Cavallo (4) hizo repuntar el PIB por habitante, en apenas cuatro años, en un porcentaje del 26.4\%, transformando a la Argentina en una de las economías más dinámicas del continente. Asimismo, en lo que hace a la estabilidad de precios, los datos no son menos elocuentes: después de convivir durante una década y media con un régimen de alta inflación, y de sufrir la dramática experiencia hiperinflacionaria de 1989 (cuando el índice de precios al consumidor llegó a ser del 4923.3\%), la Argentina alcanzó en 1994 la cifra más baja de inflación en toda su historia reciente, con un 3.6\% (5). En contraste con estos avances, el desempleo urbano ha crecido hasta tocar el $18.6 \%$, las economías regionales se debaten en una situación angustiosa y la crisis financiera desatada por el aumento en las tasas de interés en Estados Unidos, y el subsiguiente "efecto tequila", no sólo han hecho trastabillar el Plan de Convertibilidad de Cavallo, sino que también han sido ocasión para reavivar una aireada protesta social (6).

En este contexto, tres argumentos básicos contribuyen a explicar un resultado electoral anunciado (7). En primer lugar, una mayoría consistente del pais ha seguido apostando por la estabilidad (aún de su pobreza) y por la certidumbre económica de lo bueno conquistado (control de la inflación y crecimiento económico), aunque haya tenido que guardar para mejores tiempos el castigo a lo malo conocido (rezagos sociales de larga data, escándalos de corrupción, la presen-

4. Domingo Cavallo fue designado Ministro de Economía a principios de 1991 .

5. Los datos pertenecen al Balance Preliminar de la Economia de América Latina y el Caribe de la CEPAL, 1994.

6. En los últimos tiempos se han producido diversos estallidos sociales en el interior del pais, que incluyeron severos enfrentamientos entre obreros, empleados públicos y la policía. Entre los más destacados se encuentran los siguientes: Santiago del Estero, diciembre de 1993; Jujuy, 29 de marzo de 1994; Tierra del Fuego, 12 de abril de 1995; Córdoba, 23 de junio de 1995 y San Juan, 27 de julio de 1995.

7. Con un alto porcentaje de indecisos que rondaba el $27 \%$, todas las encuestas serias anticipaban un triunfo de Carlos Menem con un 32,4\%, seguido por el lider de FREPASO, José Octavio Bordón, con un 20,3\%, mientras que en tercer lugar marchaba el candidato radical Horacio Massaccesi (12,9\%). Las cifras corresponden una encuesta del CEOP (Centro de Estudios de Opinión Pública). Al proyectar la intención de los indecisos Menem obtenía cerca del $42 \%$ y Bordón el $30 \%$. Cfr. Clarin. Edición Internacional, 28 de Marzo al 3 de Abril, p. 3. 
cia de ciertos personajes en la corte presidencial que más vale perderlos que encontrarlos, etc).

En segundo término, el electorado le ha seguido restando el apoyo a las fuerzas de oposición que no han sabido descifrar un dilema perturbador: cómo constituirse en una "alternativa" de poder frente a un programa de gobierno que, en lo esencial, no tiene alternativas. Claro que decir que no tiene "alternativas" no significa que no tenga "correctivos", pero ¿corregido cómo? Después del éxito del Plan Cavallo la oposición se ha visto en el brete de navegar por aguas difíciles: no puede alterar los trazos gruesos del actual modelo, a riesgo de recaer en desequilibrios macroeconómicos inmanejables, pero tampoco puede agregar adherentes sin cuestionar las consecuencias sociales del Plan ofreciendo un programa creíble de reformas progresistas. Mientras este entuerto se resuelve, Menem parece ser el único capaz de avanzar a paso firme entre las dudas y las vacilaciones de sus adversarios, presentándose ante la sociedad $-\mathrm{y}$ en particular ante los factores reales de podercomo la garantía de la estabilidad económica del país. Si a esto le sumamos que el Frente liderado por Bordón, principal fuerza electoral de oposición en los últimos comicios, es una agrupación aluvional cuya capacidad para generar razonables condiciones de gobernabilidad estaban seriamente en duda, tendremos un cuadro más completo de las razones que impulsaron el voto en favor del candidato peronista.

Finalmente, hay otro factor que contribuye a explicar la continuidad del menemismo en el poder y que se enlaza con las raíces históricas de la inestabilidad argentina: por primera vez en muchos años, en varias décadas, la Argentina tiene un rumbo, una sensación de orden político y económico con una dirección indiscutida. Estos elementos de orden económico y rumbo político constituyen valores especialmente estimados para una sociedad que parecía condenada a vivir cien años de inestabilidad y de desasosiego. Ninguna de las otras fuerzas garantizaba, ni de lejos, estas valiosas condiciones. En buena medida, después del gobierno de Menem, y en base a la definición de estos nuevos rumbos, se acabaron ciertas discusiones, cierto tono divagatorio en torno a la definición de lo posible aunque a sus márgenes haya quedado el añorado territorio de lo deseable.

Pero si hoy por hoy es ya un lugar común decir que el gobierno de Menem ha generado una transformación profunda y duradera en la Argentina, más difícil, en cambio, es precisar las condiciones que hicieron posible esa transformación y sus perspectivas de mediano plazo. Para abordar estas cuestiones trataré de ubicar los cambios acontecidos en la Argentina en el marco comparativo de otros casos latinoamericanos.

\section{LA MULTIPLE TRANSICION}

El tiempo corto -solía decir Fernand Braudel-es la más engañosa, la más caprichosa de las duraciones. Sin embargo hay tiempos relativamente breves que en su apretada coyuntura condensan mejor que otros esas duraciones múltiples y contradictorias que nos enseñó a desentrañar el célebre historiador francés. En tal sentido, cuando observamos los cambios sociales, económicos y políticos ocurridos en Argentina y América Latina durante los últimos años, es insoslayable pensar que estamos viviendo uno de esos tiempos densos, una confluencia problemática y multifacética de varias transiciones.

En primer lugar, nos encontramos con una transición social (o societal) de largo aliento, una transición que ha permitido la emergencia de sociedades más complejas y plurales, fruto de la modernización socioeconómica y cultural de las décadas precedentes, que ya no pueden ser contenidas en los marcos estrechos de un autoritarismo vertical. Habitualmente se señala la década de los años cuarenta como el punto de arranque de un vasto proceso de urbanización, industrialización y extensión del bienestar social que bien puede ser calificado como de "modernización desarrollista". La etiqueta puede ser imperfecta, pero en todo caso sirve para destacar el hecho de que esta tendencia modernizadora de largo aliento ha sufrido en los últimos años un cambio de ritmo y de perspectiva. Así, a la dinámica de la modernización desarrollista se le ha sobreimpreso, generando tensiones y conflictos de nuevo tipo, una "modernización globalizadora" cuyos rasgos característicos son objeto de arduo debate en la actualidad. En cualquier caso, quizá el rasgo dominante de este nuevo estilo "modernizador" sea la separación del patrón de crecimiento respecto del patrón de pleno empleo, y las todavía inciertas consecuencias sobre la conformación de una nueva estructura social (8).

En segundo término, asistimos también a una transición económica, en el sentido de que los programas de ajuste y reestructuración aplicados en América latina desde finales de los años setenta y principios de los ochenta han reemplazado al viejo modelo de desarrollo de posguerra por otro basado en la sistemática liberalización del mercado y la creciente privatización e internacionalización de las economias latinoamericanas; en este proceso, a su vez, se ha venido redefiniendo el papel del Estado como regulador de la esfera económica y en su relación con actores sociales estratégicos. En el marco de esta transición económica es útil distinguir al menos tres subprocesos diferenciados: el proceso de "estabilización económica" o de ajuste en sentido restringido (equilibrio fiscal, reducción del déficit en la balanza de pagos y control de la inflación a niveles compatibles con un crecimiento continuo y sostenido); el proceso de "reforma estructural", cuyas coordenadas básicas son la apertura comercial y la desregulación de los mercados (en esta fase es donde habitualmente se produce la desincorporación o privatización de empresas públicas); y por último, el proceso de "reestructuración económica", un vasto y complejo conjunto de políticas orientados a definir el perfil productivo de un país.

Según es fama, el sentido común político y académico ha calificado a ese abigarrado panorama de cambios con el rótulo de "neoliberal". Sin embargo, es necesario hacer una distinción que más de una vez se pasa por alto, y que se refiere a la diferencia entre un "modelo económico" y los

8. El texto clásico que examina la dinámica de la "modernización desarrollista" es Gino Germani, Sociologia de la Modernización, BsAs, Paidós, 1969, esp. cap. I. En lo que se refiere a la "modernización globalizadora" vale la pena leer el sugerente trabajo de Claus Offe, “¿Empleo para todos?”, Etcétera, N.132, Agosto de 1995. 
"paquetes de políticas económicas" (9). Naturalmente, todo modelo se compone de políticas, pero mientras algunas de ellas definen la arquitectura fundamental del ordenamiento económico, otras se limitan a operar sobre zonas o arenas más específicas. De acuerdo con esto, las líneas generales de un modelo vienen definidas por dos coordenadas básicas, la modalidad de integración a la economía mundial y la relación entre Estado y mercado. Pero es importante destacar que el mismo modelo "neoliberal" puede dar lugar a notorias diferencias en el nivel de las políticas específicas: tipo de cambio, instrumentos de captación de ahorro externo, política industrial, etc.

Esta distinción elemental entre modelos y paquetes es importante por tres razones. En primer lugar, por necesidades taxonómicas: los países latinoamericanos han adoptado los rasgos básicos del "modelo" pero difieren fuertemente en el rubro de los "paquetes" específicos de políticas económicas. En segundo término, la distinción es importante por razones explicativas: el "efecto tequila" afectó a México y a la Argentina de una manera que no afectó a otros países con el mismo modelo (Chile, Perú o Brasil por caso). Y también es útil por razones heurísticas: la etapa de reestructuración que estamos viviendo trasciende largamente los dictados de las recetas neoliberales originarias, contenidas en los rasgos básicos del modelo, y se interna en una fase muchos más conjetural, donde los actores socioeconómicos y políticos han comenzado a ensayar fórmulas "correctivas" en lo que se refiere a la apertura comercial, la política industrial o a la generación de programas activos de empleo (10). Como veremos después, las configuraciones políticoinstitucionales que permitieron llevar adelante las etapas "destructivas" de la reforma económica (ajuste y cambio estructural), bien pueden en la actualidad no ser útiles para el desarrollo de la fase de reestructuracióin económica.

Finalmente, nos encontramos con la "transición politica", donde se ha acostumbrado a distinguir una serie de procesos intimamente vinculados. Por un lado, la "liberalización" política, entendido como "el proceso que vuelve efectivos ciertos derechos que protegen a individuos y grupos sociales ante los actos arbitrarios o ilegales cometidos por el Estado o por terceros" (el habeas corpus, la inviolabilidad de la correspondencia y de la vida privada en el hogar, el derecho a defenderse según el debido proceso, libertad de expresión, ausencia de censura en los medios de comunicación, libertad de asociación, etc.) (11). Por otra parte, el proceso de "transición a la democracia" o de

9. Esta distinción me viene sugerida por la lectura del trabajo de Jaime Ros, "La crisis mexicana: causas, perspectivas, lecciones", Nexos, N. ${ }^{2} 209$, Mayo de 1995 , pp.43-49.

10. Es dificil aceptar, salvo para aquellos que utilizan el término "neoliberal" como grito de guerra y no como instrumento descriptivo, que la actual politica de promoción de las exportaciones de Chile o el debate en torno a las cuotas de exportación-importación entre Argentina y Brasil en el marco del MERCOSUR puedan ser calificadas como "neoliberales". En el mismo sentido, los recientes reclamos de la Unión Industrial Argentina (UIA) por una apertura comercial "selectiva" y una politica de promoción industrial "inteligente" indican un importante cambio discursivo en lo que se refiere a la "corrección" del modelo económico vigente a partir del Plan Cavallo. Cfr. las declaraciones de Jorge Blanco Villegas, titular de la UIA, publicadas en el Suplemento Económico del diario Clarin (Edición Internacional, 27 de agosto de 1995).

11. O'Donnell, Guillermo; Schmitter, Philippe C. \& Whitehead, Lawrence, Transiciones desde un Gobierno Autoritario (1986), BsAs, Paidós, 1988, 4 vols. Cfr. esp. vol 4, cap. I, p.20 "democratización", el cual ha sido caracterizado como un cambio en el patrón legitimatorio de normas que regulan el acceso, distribución y ejercicio del poder político. Y por último, el proceso de "consolidación democrática", en el que las reglas de juego político se institucionalizan, volviéndose más sólidas, estables y previsibles (12).

Ahora bien, el diferente carácter de estas múltiples transiciones ha replanteado un tópico clásico de las ciencias sociales: el análisis de los complejos vínculos entre politica, economía y sociedad. Después de varios años en los que, tal vez por reacción frente a las antiguas visiones deterministas, se llegó a veces al extremo de "aislar" a la política de factores sociales o económicos, el péndulo de los intereses académicos viene a ubicarse en los últimos tiempos en un justo medio. A la vez que se destaca la "especificidad" de la política, aspecto que los estudios transicionales ayudaron a reivindicar en su adecuada medida, también se intenta vincularla a la dinámica de los cambios sociales, económicos y culturales (13). Como lo ha señalado Marcelo Cavarozzi, destacando las virtudes prácticas de este remozado enfoque, "la perspectiva teórica dominante durante la década de 1980 concibió la implantación y consolidación de las instituciones de la democracia política como un proceso aislado a las modalidades de la transición y orientado, asimismo, a prevenir la reemergencia del autoritarismo. En la actualidad, empero, el principal desafio no es impedir las regresiones autoritarias, sino el de reconstruir una nueva matriz de relaciones político-sociales y económicas que reemplace a aquella que se agotó durante la última década y media" (14).

Así, pues, en el laberinto de estas múltiples transiciones quisiera concentrarme en el problema de la relación entre variables político-institucionales y reforma económica. $\mathrm{Pa}$ ra facilitar la argumentación, discutiré dos tesis contrapuestas, tratando de recuperar la pertinencia analítica de cada una de ellas a la hora de examinar la experiencia argentina en una perspectiva comparativa.

\section{DEL TIPO DE REGIMEN A LA DESAGREGACION DE VARIABLES POLITICO-INSTITUCIONALES}

Según se sabe, la discusión inicial sobre las transformaciones políticas y económicas en la región estuvo centrada en las diferencias relativas entre regímenes políticos (autoritarismos y democracias) tanto para llevar adelante la reforma económica, como para definir el carácter del ajuste que pretendía aplicarse. Como lo ha referido Joan Nelson, "se ha considerado, al menos desde la década de 1970, que

2. En rigor, la transición política ha tenido dos caras, una de las cuales tiende a olvidarse con frecuencia: de un lado, hemos asistido a una transición en lo que respecta a la "forma del gobierno" (autoritarismo-democracia); de otro, hemos vivido un cambio en lo que se refiere al "grado de gobierno", y que convendria caracterizar en términos de un cambio al nivel del "paradigma de gobernabilidad".

13. Para una crítica de los diversos "reduccionismos" en la literatura de los últimos años véase Carlos H. Acuña, "El Análisis de la Burguesia como Actor Politico", Realidad Económica, Revista editada por el Instituto Argentino para el Desarrollo Económico (IADE), Buenos Aires, Nov.-Dic. de 1995, N.² 128, pp. 45-77.

14. Cavarozzi, Marcelo, "Consolidación de la democracia y reconstrucción de la economia en América Latina",en Revista de la Universidad Nacional Autónoma de México, N.. 497, Junio de 1992, p.15. 
es más probable que los gobiernos autoritarios, y no las democracias, decidan e impongan medidas económicas impopulares de estabilización y ajuste" (15).

De acuerdo con esta tesis, los gobiernos autoritarios estarían en mejores condiciones que los gobiernos democráticos para tomar medidas de ajuste más oportunas y más "duras", a la vez que estarian más capacitados para hacer planes a largo plazo. Las razones que se han dado para sostener esta posición son varias, entre ellas se destacan el hecho de que los regímenes autoritarios no están sujetos a ciclos electorales, y por tanto pueden basar sus decisiones en criterios de racionalidad económica; además, generalmente tienen una menor rotación del personal bajo su mando, lo que facilita el proceso de implementación de las medidas de ajuste; adicionalmente, los regímenes autoritarios tienen menor necesidad de responder a las presiones políticas de la sociedad, e incluso aparecen como más capaces de "aislar" a los tomadores de decisiones de los grupos afectados por las decisiones del ajuste; finalmente, los regímenes autoritarios, a diferencia de las democracias, en particular las de transición reciente, pueden detener las protestas a través de la represión anticipada, y acabar con ellas si ocurrieran.

Es importante hacer notar que la versión estilizada de esta posición, que podríamos llamar la tesis StallingsKaufman (16), nada dice sobre el "éxito" final de los autoritarismos, o los fracasos de las democracias, en lo que hace al rendimiento económico post-ajuste. La razón es clara: el éxito de una economía depende de tantos factores, estructurales y coyunturales, que no es posible evaluar el impacto específico del tipo de régimen sobre el resultado final de la reforma económica. Lo que afirma la tesis, más bien, es que distintos tipos de regímenes políticos (la variable “independiente" del análisis) tenderán a optar por diferentes tipos de ajuste económico (variable "dependiente"), definidos éstos en base a tres dimensiones: oportunidad, alcance y contenido de las reformas.

Así, los regímenes autoritarios se inclinarán a tomar medidas de ajuste ante los primeros signos de crisis, mientras que los democráticos postergarán la decisión de suministrar "medicinas amargas" hasta que los tiempos electorales sean propicios; los primeros se inclinarán por una reforma económica de largo alcance (que incluye reformas "estructurales" tales como privatizaciones, desregulación de la economía, apertura comercial, etc), mientras las democracias se concentrarán en solucionar los problemas de corto plazo, es decir, aquéllos que tienen un impacto electoral inmediato (como bajar la tasa de inflación); y por último, los regímenes autoritarios son proclives a optar por un ajuste de contenido "ortodoxo" (como el que llevaron adelante Chile y México), mientras las democracias, especialmente aquellas no consolidadas, se inclinarán por programas "heterodoxos" que no amenacen la capacidad de consumo de vastos sectores de la población (el Plan Austral argentino, el Plan Cruzado

15. Joan Nelson (edit.), Crisis Económica y Politicas de Ajuste (Princeton University Press, 1990), Colombia, Norma, 1994, cap. I, p.51.

16. Cfr. Robert Kaufman y Barbara Stallings, "Debt and Democracy in the 1980's: The Latin American Experience", en Barbara Stallings y Robert Kaufman, eds., Debt Democracy in Latin America, Boulder, Colorado, Westview, 1988, pp. 201-223
Brasileño y el Plan Inti peruano serian ejemplos de este tipo de enfoque) (17).

Ahora bien, el enfoque inicial de Stallings-Kaufman abrió la ruta para investigaciones más puntuales y especificas. De este modo, la variable "tipo de régimen" pronto se reveló demasiado general para explicar comportamientos nacionales diferenciados, puesto que aún bajo la misma categorización de Estados autoritarios, como fue el caso de Pinochet en Chile y la Junta Militar que gobernó la Argentina entre 1976-1983, el carácter de las reformas económicas guardó sensibles diferencias. En rigor, hablar en general de "regímenes autoritarios" oculta las sutiles pero importantes desemejanzas entre distintas formaciones politicas, y los diferentes legados que heredaron las jóvenes democracias del subcontinente latinoamericano. Como ha señalado $\mathrm{Ka}$ ren Remmer los "académicos pasaron de estudiar la quiebra de las democracias a las transiciones democráticas sin tomarse una pausa para analizar la fase autoritaria que hubo entre ellas" (18). De acuerdo con esto, autores como Remmer se propusieron el doble objetivo de evaluar el impacto de los regímenes políticos sobre las políticas y la política de las sociedades latinoamericanas, por un lado, y el de explorar las causas de la variación en la efectividad de esos regimenes, por el otro. En este último caso, Remmer utiliza como variables privilegiadas la organización militar interna y la penetración militar de las instituciones gubernamentales. Sus ejemplos extremos son el "régimen militar-feudal" argentino (1976-1983), caracterizado por la dispersión de poder y la superposición de funciones políticas y militares que terminó politizando y diviendo en exceso a los propios militares, y el "régimen militar-sultanístico" de Pinochet (1973-1989), el cual tendió a concentrar el poder y a aislar a los tomadores de decisiones de las presiones de los diferentes grupos sociales (19). Según estos estudios, pues, serían variables político-institucionales más específicas, y no la consideración general de los "autoritarismos" vs. las "democracias", lo que explicaría los itinerarios divergentes de las reformas económicas en América Latina (20).

17. En rigor, la tesis Stallings-Kaufman también contempla la situación, junto con los regimenes autoritarios, de las democracias "consolidadas", pero en este último caso los ejemplos latinoamericanos son divergentes: mientras Colombia inició tempranamente un proceso de ajuste ortodoxo y sostenido, Venezuela retardó la decisión e incluso recayó en políticas económicas expansivas bajo el segundo gobierno de Carlos Andrés Pérez. El caso de Costa Rica es más complejo, y podría decirse que el proceso de ajuste se inició recién durante el último gobierno socialcristiano del presidente Calderón (1990-1994). Por su parte, el candidato liberacionista que ganó las últimas elecciones, José María Figueres, lo hizo con un discurso "populista" y contrario al llamado "ajuste neoliberal", pero en el primer semestre de 1995, ante el resultado de un déficit fiscal abultado pacté con sus antiguos adversarios un programa de ajuste bajo la tutela del FMI.

18. Citado por Frances Hagopian, "After Regime Change. Authoritarian Legacies, Political Representation, and the Democratic Future of South America", World Politics, Vol. 45, April 1993, N.ํ 3, pp. 464-500. La cita se encontrará en la p.466. 1989

19. Karen Remmer, Military Rule in Latin America, Boston, Unwin Hyman,

20. La recuperación analitica del papel del Estado y de sus instituciones, frente a enfoques politológicos de alta agregación (régimen o sistema politico) o sociológicos de carácter determinista, marca una tendencia pronunciada en la ciencia politica de la última década. Estos trabajos, que caen bajo la denominación de enfoques "estado-céntricos", o en términos más generales "neoinstitucionalistas", puede ser identificados, entre otros autores, con Theda Skocpol, "El Estado Regresa al Primer Plano" (Cambridge University Press, 1985), en Edelberto Torres-Rivas, Politica. Teoria y Métodos, San José de Costa Rica, EDUCA-CSUCA, 1990, y con Peter Evans "The State as problem and Solution: Predation, Embedded Autonomy, and Structural Change", Capitulo 3 de la obra ya citada de Stephan Haggard and Robert R. Kaufman, pp.139-181. Para el caso argentino puede consultarse el trabajo de Kathryn Sikkink, "Las capacidades y la autonomia del Estado en Brasil y la Argentina. Un enfoque neoinstitucionalista", Desarrollo Económico, vol. $32, \mathrm{~N}^{\circ}{ }^{\circ} 128$, enero-marzo 1993, 543-574. 
En esta misma línea, los trabajos publicados a finales de los años 80 y en los primeros años de la década de 1990 por Joan Nelson, Stephen Haggard y el propio Robert Kaufman, dieron una vuelta de tuerca adicional a la cuestión (21). En estos trabajos los autores buscaron esclarecer los factores políticos que moldearon las elecciones de los ajustes de los gobiernos y la ejecución de dichas preferencias. La hipótesis de partida era que, si bien diferentes países se encontraron con un conjunto común de condicionamientos externos desde mediados de los setenta (shokcs petroleros y dramáticos incrementos del flujo de "petrodólares", subida en las tasas de interés internacionales, caída en los términos del intercambio de productos primarios), las diferencias en lo que se refiere a la oportunidad, el alcance y el contenido de los procesos de ajuste debían explicarse, no por variables puramente económicas, sino por factores de naturaleza política.

A juicio de estos autores, cinco amplios conjuntos de factores causales servirían para explicar las orientaciones diferenciales de esos procesos de ajuste, a saber:

a) Naturaleza de la crisis económica: su severidad, aparición gradual o repentina, duración prolongada o corta, y cómo fue interpretada, en especial su persistencia esperada y las raices percibidas, ya sean principalmente externas 0 debido en gran parte a problemas domésticos.

b) Capacidad del Estado-capacidades técnicas y administrativas del Estado: en especial la capacidad técnica y la unidad del equipo económico, y la profundidad de la capacidad gerencial.

c) Estructuras politicas: tipo de régimen y variación en instituciones políticas más específicas, en particular ciclos electorales y la autonomía del ejecutivo en jefe.

d) Circunstancias politicas: liderazgo y coaliciones de apoyo y oposición.

e) Papel de las entidades extranjeras: incluyendo las instituciones financieras internacionales, gobiernos de países acreedores y acreedores comerciales (22).

De este modo, la consideración inicial en términos del "tipo de régimen" dio paso a una elaboración más detallada -que para simplificar llamaré la tesis Nelson- donde se desagregan una serie de factores políticoinstitucionales que jugaron un papel decisivo en la orientación de la reforma económica. De este cúmulo de factores y de actores que mediaron la relación entre la política y la transformación económica a lo largo de los ochenta, los autores destacan una serie de elementos preponderantes. A la hora de estudiar los casos en los cuales se adoptaron y en gran medida se implantaron reformas amplias, Joan Nelson destaca el peso, por un lado, de "la percepción generalizada de la necesidad de amplias reformas (derivada de un legado de declinación económica y debilitamiento político u oposición encarnizada)", y, por el otro, señala el papel jugado por "un poder ejecutivo fortalecido por una combinación de instituciones po-

21. Me refiero al ya citado libro de Nelson, y a los otros dos volúmenes compilados en común, a saber: Joan Nelson (edit.), Coaliciones Frágiles: La politica de ajuste económico (New Brunswick, Transaction Books, 1989), México, CE MLA/Banca Serfin y el libro de Stephen Haggard \& Robert Kaufman, The Politics of Economics Adjustment, Princeton University Press, 1992.

22. Joan Nelson, Crisis Económica..., pp. 45 y 554 líticas establecidas y circunstancias transitorias con una insólita concentración de autoridad" (23).

Asimismo, al examinar el rol jugado por diferentes actores en el proceso de reforma económica, los autores citados señalan que "el papel de las instituciones externas, aunque importante en la consideración detallada de casos particulares, es menos importante que estos factores esencialmente internos para determinar la elección y ejecución de medidas de ajuste" (24). En particular, los dos actores estratégicos que tendieron a moldear el proceso de ajuste fueron los intereses empresariales organizados y la maquinaria estatal en sí misma; mientras que los movimientos sindicales y los intereses agrícolas desempeñaron papeles mucho más modestos.

\section{EL AJUSTE EN LOS TIEMPOS DE MENEM}

Pues bien, si con estos cristales analíticos consideramos las transformaciones económicas lideradas por Carlos Menem en la Argentina, veremos la preponderancia de los factores explicativos destacados por la tesis Nelson. Conviene destacar que el interés teórico y metodológico de analizar las reformas menemistas a la luz del enfoque propuesto por Nelson y otros autores es particularmente relevante, por el hecho de que estos trabajo no incluyen en su examen el período de gobierno de Menem. De acuerdo con esto, el caso argentino serviría para efectuar una suerte de "corroboración" ex-post del planteo analítico propuesto.

En efecto, el estado social generado por la hiperinflación que sufrió la Argentina a mediados de 1989 y el ejercicio eficaz y concentrado del poder político en manos del nuevo gobierno han sido reconocidos como los dos pilares fundamentales que permitieron llevar adelante las reformas económicas bajo la administración de Carlos Menem.

En lo que hace al primer factor podemos hablar, siguiendo a Juan Carlos de Pablo, de la herencia económica "mediata" y la herencia "inmediata" que recibió Menem al asumir la presidencia el 8 de julio de 1989, cinco meses antes de lo que fijaba el plazo constitucional (25). La herencia mediata se ubica (26) en el legado de los años ochenta, que la CEPAL ha calificado como la "década perdida", en la cual la economía argentina sufrió un retroceso del PIB per cápita de $-21.2 \%$ (entre 1981 y 1990) y una inflación promediada para esos diez años del $876.01 \%$ anual. La herencia inmediata, por su parte, se resume en la dramática experiencia hiperinflacionaria de 1989, cuando el índi-

23. Ibidem, p. 65.

24. Ibidem, p. 66.

25. Alfonsin debia entregar el poder a Menem el 10 de diciembre de 1989, al cumplir seis años de mandato presidencial. Pero en medio de la situación de ingobernabilidad generada por la hiperinflación, y después de febriles e infructuosas conversaciones entre los delegados de ambos líderes políticos, el presidente saliente anuncio en un discurso a la nación el 12 de junio que "resignaba" su investidura presidencial: finalmente, se decidió que el 8 de julio asumiria el nuevo presidente electo. Los entretelones de estas negociaciones se encontrarán en Luis Majul, POR QUE CAYÓ ALFONSÍN. El nuevo terrorismo económico, Buenos Aires, ed. Sudamericana, 1990, sexta parte: y también en el libro de Gabriela Cerruti, EL JEFE. Vida y obra de Carlos Saúl Menem, Buenos Aires, Planeta, 1993, cap. 8

26. El análisis de la herencia económica "lejana" nos llevaria a examinar el comportamiento de la economía argentina y de sus agentes económicos desde los años cuarenta, asunto que dejamos fuera de nuestro análisis. 
ce de precios al consumidor llegó a ser del 4923.3\%. Pero como señala de Pablo, "una hiperinflación no es sólo un aumento vertiginoso de la tasa de inflación, es principalmente la horrible sensación de no saber qué va a pasar, ni cómo ni cuándo va a terminar el terremoto. El horizonte de decisiones se reduce al día en que se vive y por consiguiente se limita a las necesidades biológicas. Mucha gente que en circunstancias normales es tenaz se paraliza, porque si bien entiende lo que sucede, el terror no le permite actuar. Buena parte de la historia que se cuenta en esta obra no habría sucedido si previamente los argentinos no hubiéramos pasado por una híper" (27). De este modo, la experiencia hiperinflacionaria, sumada a la percepción social de un comportamiento negativo de la economía por varios años, abrió el camino para que la nueva administración implementara un programa de estabilización y de reforma estructural de vasto alcance y de naturaleza fuertemente ortodoxa.

Por otra parte, el "acuerdo" por el traspaso adelantado del poder contemplaba que el gobierno saliente facilitaría "la inmediata sanción de las leyes económicas que el presidente futuro considere necesarias" (28). Este acuerdo permitió al gobierno sancionar dos "super" leyes que constituyeron la estructura jurídica fundamental para llevar adelante el programa de ajuste, se trata de la ley de "Reforma del Estado" (17 de Agosto) y la ley de "Emergencia Económica" ( 1 de Septiembre) que dotaban al poder ejecutivo de amplias atribuciones para decidir privatizaciones, suspensión de subsidios y contrataciones, disponibilidad de empleados públicos, etc.

Asimismo, este fortalecimiento jurídico transitorio, pero efectivo, del poder ejecutivo nacional se vio acrecentado tanto por las condiciones de liderazgo político que ejercía Menem al interior del peronismo, como por su particular estilo de gobierno. En el primer aspecto hay que señalar que el nuevo presidente ejercía un tipo de liderazgo inorgánico, fundado en su carisma personal, y alejado tanto de las estructuras partidistas como distanciado de la conducción sindical oficial. Además, un estilo de gobierno audaz y decidido, que le llevó en múltiples ocasiones a apelar a mecanismos de decisión "extraordinarios" (que pasaban por encima de las atribuciones del Congreso) para lograr sus propósitos reformistas (29), completan este cuadro de autonomía e insulación del poder ejecutivo menemista. Por aquello que señalaba Maquiavelo, según el cual "la reforma de los Estados corrompidos o la creación de otros nuevos debe ser obra de un solo hombre" (30), Menem tuvo claro desde el principio de su gobierno, que para llevar a cabo la transformación económica que se

27. Juan Carlos de Pablo, QUIEN LO HUBIERA DICHO. La transformación que lideraron Menem y Cavallo, Buenos Aires, Planeta, 1994, p. 16 y 17

28. Luis Majul, p. 232. La razón de esto es que si bien se adelantaba el traspaso del poder ejecutivo, el gobierno entrante no dispondría de sus propios legisladores hasta el 10 de diciembre de 1989.

29. El mecanismo de referencia son los llamados "decretos de necesidad y urgencia" cuyo rango constitucional ha sido objeto de larga controversia. Una compración (realizada por Mateo Goretti et al.) resultará elocuente para contrastar el estilo de gobierno de Menem con el de su predecesor: mientras Alfonsin promulgó en cinco años y medio de administración unos 10 decretos de esta naturaleza, Menem promulgó -desde Julio de 1989 a Diciembre de 1992- 244, la mayoría de los cuales se referian a cuestiones en materia económica. Cfr. Carlos H. Acuña, La Matriz..., Nota 62 al cap. 8

30. Citado por Samuel PHuntington, El Orden Politico en las Sociedades en Cambio, Buenos Aires, Paidós, 1972, p. 215 proponía, debía contar con una amplia libertad de movimientos y una clara orientación en lo que respecta al sentido de las reformas (31).

No obstante esto, se hace necesario hoy tomar alguna distancia respecto de la solidez, o la fragilidad potencial, de las transformaciones encaradas. Como señalaba alguna vez Albert O. Hirschman, la realidad tiene una endiablada forma de ser según la cual "las soluciones de una etapa suelen constituirse en los problemas de la otra". La moraleja de este aserto puede ser apreciada en la actualidad toda vez que las mismas condiciones que hicieron posible el rápido cambio de orientación económica del país (situación crítica generada por la hiperinflación, concentración de poder no sujeto al control efectivo del Congreso y el liderazgo inorgánico de Menem), se tradujeron también en una implementación severamente desproiija del contenido de las reformas estructurales (32) y en una debilidad de origen en lo que respecta al proceso de gestión en el postajuste, es decir, el proceso de re-regulación del Estado sobre el campo ampliado de las actividades privadas. Esto último es especialmente notorio en lo que se refiere a la regulación del funcionamiento de las empresas estatales que pasaron a la órbita privada, donde existen numerosos "vacios" jurídicos en lo referente al control de prácticas monopólicas o a la exigencia de standars adecuados en la provisión de servicios públicos.

Por otra parte, y este asunto tal vez tenga una complejidad mayor que el anterior, el proceso de ajuste ha dejado abierto el desafio de garantizar las condiciones suficientes para un desarrollo sostenido. En tal sentido, si el proceso inicial de reforma económica requirió un Estado con un poder concentrado y capaz de tomar las duras decisiones del ajuste, en la actualidad parece necesario la conformación de un aparato estatal sensible a la colaboración con los sectores productivos.

De este modo, en los últimos años un conjunto de autores han abogado por una redefinición de las relaciones entre Estado, mercado y sociedad civil, que supere los extremos fáciles del viejo modelo del Estado interventor o los limitados horizontes del llamado Estado "mínimo". En esta línea se destaca la necesidad de que el Estado desarrolle una estructura de "autonomía imbricada" con el mercado y la sociedad, en la que se combinen la "coherencia interna" del aparato estatal (dotado de una burocracia eficiente, un grado significativo de autonomía respecto de los intereses inmediatos de los grupos sociales circundantes, una concentración del saber técnico, un reclutamiento meritocrático y la provisión de oportunidades mediante una carrera bien remunerada de largo plazo) con la "conectividad externa" respecto de sectores económicos, grupos y organizaciones de la sociedad civil, para permitir un intercambio productivo en el proceso de formulación e implementación de las politicas económicas y sociales.

31. Pasamos por alto en esta breve reseña el papel de los distintos actores politicos y sociales, y el modo en que el menemismo logró superar oposiciones y conformar coaliciones de apoyo a su politica. Para un tratamiento detallado de estos puntos puede verse el trabajo de Acuña citado en último lugar.

32. El tema de la corrupción ha venido llenando innumerables páginas en la $\mathrm{Ar}$ gentina de los últimos años, me limito a señalar un "clásico" de esa dispar literatura: Horacio Verbitsky, Robo para la Corona, Buenos Aires, Planeta, 1991. 
Como ha señalado Peter Evans, las tareas del desarrollo requieren algo más (o algo distinto) que un Estado autónomo y concentrador del poder; más bien, se necesitan agencias estatales activas que posean conexiones fluidas con el capital privado para garantizar la fase de implementación de políticas. En palabras del propio Evans, la capacidad para transformar la economía requiere una combinación de coherencia interna del aparato estatal y de conectividad externa con el mercado y los grupos sociales y económicos estratégicos, de modo tal de configurar un nuevo tipo de Estado que posea una "embedded autonomy" (33).

Quizá en el logro de estas nuevas tareas, una reregulación del mercado en el período del post-ajuste y una renovada capacidad estatal para llevar adelante los requerimientos de un desarrollo sostenido crecientemente equitativo, se encuentren algunos de los más importantes desafios de la recuperada democracia argentina.

\section{RESUMEN}

Antonio Camou realiza un repaso a la evolución política argentina que va de la presidencia de Alfonsín a la de Menem, señalando los que considera los móviles fundamentales del apoyo electoral recibido por el peronismo en las últimas elecciones. Posteriormente, examina los cambios políticos y económicos experimentados por Argentina en la última década en una perspectiva comparada con el resto de países latinoamericanos, analizando la vinculación entre variables político-institucionales y reforma económica.

ABSTRACT
Antonio Camou makes a review of the Argentinian political evolution during the period from the
Alfonsin's presidence to Menem's presidence. He points out what he considers the mean reasons of
menemism electoral support in recent polls. Later, he examines political and economical changes
suffered by Argentina in the last decade in a comparative perspective with the other Latin American
countries. He analyzes the links between political-institutional variables and economical reform.

33. Peter Evans, "The State as problem...", p. 176. 